\title{
AN INTEGRAL CRITERION FOR NORMAL FUNCTIONS
}

\author{
RAUNO AULASKARI AND PETER LAPPAN
}

(Communicated by Irwin Kra)

\begin{abstract}
A new characterization for normal functions is given. It is shown that a function $f$ meromorphic in the unit disk is a normal function if and only if for each $\delta>0$ and each $p>2$ there exists a constant $K_{f}(\delta, p)$ such that, for each hyperbolic disk $\Omega$ with hyperbolic radius $\delta$,

$$
\iint_{\Omega}\left(1-|z|^{2}\right)^{p-2}\left(f^{\#}(z)\right)^{p} d A(z) \leq K_{f}(\delta, p),
$$

where $f^{\#}(z)$ denotes the spherical derivative of $f$ and $d A(z)$ is the Euclidean element of area. It is shown by example that this characterization is not valid for $p=2$.
\end{abstract}

Let $D=\{z:|z|<1\}$ denote the unit disc, let $\rho\left(z_{1}, z_{2}\right)$ denote the hyperbolic distance between the points $z_{1}$ and $z_{2}$ in $D$, and let $m_{D}(\Omega)$ denote the hyperbolic area of the subset $\Omega$ of $D$. For a function $f$ meromorphic in $D$, let the spherical derivative of $f$ be denoted by

$$
f^{\#}(z)=\frac{\left|f^{\prime}(z)\right|}{1+|f(z)|^{2}} .
$$

A function $f$ meromorphic in $D$ is said to be a normal function if

$$
\sup \left\{\left(1-|z|^{2}\right) f^{\#}(z): z \in D\right\}<\infty .
$$

There are a number of known characterizations of normal functions. (See, for example, $[1,2,4$, and 5].)

In this note, we give a new characterization of a normal function. This characterization bears a similarity to a result of S. Yamashita [7] characterizing Bloch functions.

THEOREM. A function $f$ meromorphic in the unit disc is a normal function if and only if, for each $\delta>0$, and each $p>2$, there exists a constant $K_{f}(\delta, p)$, such that, for each hyperbolic disc $\Omega$ with hyperbolic radius $\delta$,

$$
\iint_{\Omega}\left(1-|z|^{2}\right)^{p-2}\left(f^{\#}(z)\right)^{p} d A(z) \leq K_{f}(\delta, p),
$$

where $d A(z)$ denotes the element of Euclidean area.

PROOF. Suppose that $f$ is a normal function. Then we have that there exists a constant $C$ such that for each $z \in D,\left(1-|z|^{2}\right) f^{\#}(z) \leq C$. Further, we note that

$$
d m_{D}(z)=\frac{d A(z)}{\left(1-|z|^{2}\right)^{2}}
$$

Received by the editors March 3, 1987 and, in revised form, May 5, 1987.

1980 Mathematics Subject Classification (1985 Revision). Primary 30D45. 
and so the integral $\iint_{\Omega}\left(1-|z|^{2}\right)^{p-2}\left(f^{\#}(z)\right)^{p} d A(z)$ is the same as the integral $\iint_{\Omega}\left(\left(1-|z|^{2}\right) f^{\#}(z)\right)^{p} d m_{D}(z)$. This last integral is clearly dominated by $C^{p} m_{D}(\Omega)$, which is finite, since both $C^{p}$ and $m_{D}(\Omega)$ are finite. Clearly, $C^{p} m_{D}(\Omega)$ depends only on $f, p$, and the radius of $\Omega$. This proves the "only if" part of the theorem. (Note that the result holds in this direction for each positive $p$.)

Now suppose that $f$ is not a normal function and that $p>2$ and $\delta>0$ are fixed. Then, by a result of Lohwater and Pommerenke [6, Theorem 1, p. 3] there exist a sequence of points $\left\{z_{n}\right\}$ in $D$ and a sequence of positive numbers $\left\{p_{n}\right\}$ such that $p_{n} /\left(1-\left|z_{n}\right|\right) \rightarrow 0$ and the sequence of functions $\left\{f_{n}(t)=f\left(z_{n}+p_{n} t\right)\right\}$ converges uniformly on each compact subset of the complex plane to a nonconstant meromorphic function $g(t)$. For each $n$, let $\Omega_{n}=\left\{z: \rho\left(z, z_{n}\right)<\delta\right\}$. Let $s>0$ be fixed. Then for $n$ sufficiently large, since $p_{n} /\left(1-\left|z_{n}\right|\right) \rightarrow 0$, the set $\Gamma_{n}=$ $\left\{z: z=z_{n}+p_{n} t,|t|<s\right\} \subset \Omega_{n}$. We note that $f_{n}^{\prime}(t)=p_{n} f^{\prime}\left(z_{n}+p_{n} t\right)$, and that $d A(z)=\left(p_{n}\right)^{2} d A(t)$. Thus, $\iint_{\Omega_{n}}\left(1-|z|^{2}\right)^{p-2}\left(f^{\#}(z)\right)^{p} d A(z)$ is larger than the integral $\iint_{\Gamma_{n}}\left(1-|z|^{2}\right)^{p-2}\left(f^{\#}(z)\right)^{p} d A(z)$, and, if we convert this to an integral where the variable of integration is $t$, we get

$$
\iint_{|t|<s}\left(\frac{1-\left|z_{n}+p_{n} t\right|^{2}}{p_{n}}\right)^{p-2}\left(f_{n}^{\#}(t)\right)^{p} d A(t) .
$$

But

$$
\iint_{|t|<s}\left(f_{n}^{\#}(t)\right)^{p} d A(t) \rightarrow \iint_{|t|<s}\left(g^{\#}(t)\right)^{p} d A(t),
$$

and this last integral is finite and nonzero, since $g(t)$ is a nonconstant meromorphic function and the integral is over a fixed bounded set. However, since $p_{n} /\left(1-\left|z_{n}\right|\right) \rightarrow$ 0 we have that $\left(1-\left|z_{n}+p_{n} t\right|\right) / p_{n} \rightarrow \infty$ uniformly for $|t|<s$, and it follows that

$$
\iint_{\Gamma_{n}}\left(1-|z|^{2}\right)^{p-2}\left(f^{\#}(z)\right)^{p} d A(z) \rightarrow \infty .
$$

This proves the "if" part of the theorem and completes the proof.

We now show that the theorem is not valid for $p=2$. Let $f(z)$ be a locally uniformly univalent function which is not a normal function (see Lappan [3]). Let $\beta>0$ be such that the function $f(z)$ is univalent in any disc of hyperbolic radius $\beta$. Then for each $w \in D$, if $\Lambda(w)=\{z: \rho(z, w)<\beta\}$ we have that $f(z)$ is univalent in $\Lambda(w)$ and hence the spherical area of $f(\Lambda(w))$ is less than $\pi$, that is,

$$
\iint_{\Lambda(w)}\left(f^{\#}(z)\right)^{2} d A(z)<\pi
$$

Now let $\Omega$ be any hyperbolic disc in $D$ with finite radius $\delta>0$. Then there exists a finite number of points $w_{1}, w_{2}, \ldots, w_{k}$ such that $\Omega \subset \bigcup_{j=1}^{k} \Lambda\left(w_{j}\right)$. It follows that

$$
\iint_{\Omega}\left(f^{\#}(z)\right)^{2} d A(z) \leq k \pi
$$

where $k$ depends only on $\delta$. Thus, the Theorem is not valid for $p=2$.

\section{REFERENCES}

1. P. M. Gauthier, A criterion for normalcy, Nagoya Math. J. 32 (1968), 277-282.

2. V. I. Gavrilov, On the distribution of values of functions meromorphic in the unit circle which are not normal, Mat. Sb. 67 (1965), 408-427. (Russian) 
3. P. Lappan, A non-normal locally uniformly univalent function, Bull. London Math. Soc. 5 (1973), 291-294.

4. __ A criterion for a meromorphic function to be normal, Comment. Math. Helv. 49 (1974), 492-495.

5. O. Lehto and K. I. Virtanen, Boundary behaviour and normal meromorphic functions, Acta Math. 97 (1957), 47-65.

6. A. J. Lohwater and Ch. Pommerenke, On normal meromorphic functions, Ann. Acad. Sci. Fenn. Ser. AI, No. 550 (1973), 12 pp.

7. S. Yamashita, Criteria for functions to be Bloch, Bull. Austral. Math. Soc. 21 (1980), 223-227.

Department of MAthematics, University of JoensuU, SF 80101 JoensuU 10 , FINLAND

Department of Mathematics, Michigan State University, East Lansing, MICHIGAN 48824 